\title{
Mapeamento de Chuvas Intensas para o Estado do Tocantins
}

\author{
Virgilio Lourenço Silva Neto ${ }^{1}$ (D), Marcelo Ribeiro Viola ${ }^{2}$, Carlos Rogério Mello ${ }^{2}$, \\ Marcos Vinicius Giongo Alves ${ }^{3}$, Demetrius David Silva ${ }^{4}$, Silvio Bueno Pereira ${ }^{4}$ \\ ${ }^{1}$ Instituto Federal de Educação, Ciências e Tecnologia do Tocantins, Dianópolis, TO, Brasil. \\ ${ }^{2}$ Universidade Federal de Lavras, Departamento de Recursos Hidricos e Saneamento, Lavras, \\ $M G$, Brasil. \\ ${ }^{3}$ Universidade Federal do Tocantins, Programa de Pós-Graduação em Ciências Florestais e \\ Ambientais, Gurupi, TO, Brasil. \\ ${ }^{4}$ Departamento de Engenharia Agrícola da Universidade Federal de Viçosa, MG, Brasil.
}

Recebido em: 16 de Fevereiro de 2018 - Aceito em: 7 de Outubro de 2019

\begin{abstract}
Resumo
Este estudo teve como objetivo mapear chuvas intensas com durações de 10, 30, 720 e 1440 minutos, associadas aos tempos de retorno de 10, 50 e 100 anos, para o Estado do Tocantins. Foram aplicadas séries históricas de 95 postos pluviométricos localizados no Estado do Tocantins e proximidades, com período padronizado de 1983 a 2013. Foi conduzido um estudo geoestatístico, avaliando-se os modelos de semivariograma esférico, exponencial e gaussiano, ajustados pelo método dos mínimos quadrados ponderados. A partir deste estudo, o modelo que apresentou o menor erro médio com base na validação cruzada foi aplicado ao processo de mapeamento por krigagem ordinária. Houve bom desempenho das técnicas geoestatísticas, podendo-se destacar o modelo esférico para o mapeamento da precipitação máxima diária anual, e o modelo gaussiano para as chuvas calculadas pela metodologia de desagregação. Verificou-se ainda que as áreas de ocorrência das chuvas intensas de maior severidade no estado estão no Bico do Papagaio (norte), Ilha do Bananal (sudoeste) e noroeste do estado.
\end{abstract}

Palavras-chave: chuva de projeto, desagregação de chuvas, geoestatística.

\section{Heavy Rainfall Mapping for Tocantins State, Brazil}

\begin{abstract}
This study aimed to map heavy rainfall with durations of 10, 30, 720 and 1440 minutes, associated with return times of 10, 50 and 100 years for the Tocantins State. Historical series of 95 pluviometric stations located in the Tocantins State and nearby were applied, with standard period from 1983 to 2013. A geostatistical study was conducted, evaluating the spherical, exponential and gaussian semivariogram models, adjusted by the weighted least squares method. From this study, the model that presented the smallest average error based on cross-validation was applied to the mapping process by ordinary kriging. There was good performance of the geostatistical techniques, being possible to highlight the spherical model for the mapping of the annual maximum daily precipitation and the Gaussian model for the rains calculated by the methodology of disaggregation. It was also verified that the areas of intense rainfall of greater severity in the state are in the Bico do Papagaio (north), Bananal Island (southwest) and northwest of the state.
\end{abstract}

Keywords: rainfall design, rainfall breakdown, geostatistics.

\section{Introdução}

Chuva intensa é todo evento de chuva cuja lâmina precipitada ou sua intensidade supere um valor mínimo que é função do tempo de duração da chuva (Melo e Silva,
2013). Segundo Silva et al. (2002), o conhecimento da equação que relaciona intensidade, duração e frequência das chuvas intensas, apresenta grande interesse de ordem técnica nos projetos de obras hidráulicas, como dimensio-

Autor de correspondência: Virgilio Lourenço Silva Neto, virgilio.neto@ifto.edu.br. 
namento de vertedores, retificação de cursos d'água, galerias de águas pluviais, bueiros, sistemas de drenagem agrícola, urbana e rodoviária, dentre outros.

Para o planejamento integrado da gestão de recursos hídricos e do uso do solo é fundamental compreender a dinâmica espaço-temporal das chuvas intensas sob uma perspectiva ampla. Isto é importante para fornecer subsídios para o dimensionamento de projetos de conservação dos solos e de obras hidráulicas, e também para o planejamento do uso e ocupação da paisagem, visto que o conhecimento das características das chuvas intensas subsidia a identificação de áreas de risco para ocupação humana (Mello e Viola, 2013).

As chuvas intensas são caracterizadas por sua intensidade (I), duração (D) e frequência de ocorrência (F), sendo frequentemente representadas por equações que modelam a relação entre estas três grandezas, para determinado local (Souza et al., 2012). A determinação das equações IDF por meio de dados pluviográficos apresenta grande dificuldade em razão da baixa densidade da rede pluviográfica e do pequeno período de observações normalmente disponível (Back et al., 2011). Por esta razão, em algumas regiões poucos trabalhos têm sido realizados com tal finalidade ocasionando grande entrave na realização de projetos de obras hidráulicas mais confiáveis e econômicos (Souza et al., 2012).

Desde o estudo pioneiro sobre chuvas intensas no Brasil desenvolvido por Pfafstetter (1957), diversos aperfeiçoamentos foram realizados, podendo-se destacar os estudos de Festi (2007), que elaborou uma coletânea de equações de chuvas intensas, Silva et al. (2002) para o Estado da Bahia, Souza et al. (2012) para o Estado do Pará, Oliveira et al. (2000) e Aragão et al. (2013) para o Estado do Sergipe. Especificamente para o Estado do Tocantins, Silva et al. (2003) elaboraram equações de IDF para 10 localidades, Marcuzzo e Goularte (2012) determinaram índices de anomalias de chuvas e Viola et al. (2014) estudaram o comportamento do Fator R (erosividade) da equação universal de perdas do solo (USLE).

A importância do mapeamento das grandezas climáticas consiste tanto na busca por melhorias de aspectos metodológicos quanto na geração de mapas de uso e aplicação prática para o planejamento do meio físico, manejo de bacias hidrográficas e projetos de obras hidráulicas. Neste tipo de estudo, a utilização de técnicas geoestatísticas possibilita a redução de erros aleatórios, uma vez que a dependência espacial da precipitação é levada em consideração, o que permite o controle da parcela deste erro associada à dependência espacial (Mello et al., 2003).

$\mathrm{O}$ Estado do Tocantins encontra-se em uma região de transição climática, no contato entre os Biomas Cerrado e Amazônia, e relativamente próximo do semiárido do Nordeste do Brasil. O regime de chuvas sofre influência, dentre outros, do bloqueio atmosférico Anticiclone do Atlântico Sul (ACAS) e da Zona de Convergência do
Atlântico Sul (ZCAS). O regime pluvial é caracterizado pela marcante sazonalidade da região central do Brasil, na qual, durante o período de estiagem, ocorre a intensificação do anticiclone do Atlântico Sul, com redução da presença de vapor d'água na atmosfera, o que impede a organização de nuvens de chuva e o deslocamento de sistemas frontais (Reboita et al., 2010).

Durante os meses de outubro a abril ocorre cerca de 90\% da precipitação total anual no Estado do Tocantins (Marcuzzo e Goularte, 2013), prevalecendo eventos convectivos, que são caracterizados por elevada intensidade, curta duração e elevado potencial erosivo. Nesse contexto, torna-se essencial o desenvolvimento de pesquisas detalhadas que subsidiem o reconhecimento da distribuição espacial e temporal das chuvas intensas nas distintas regiões ecológicas presentes no estado do Tocantins, visando ao planejamento de práticas de manejo e de conservação do solo e da água, projetos de obras hidráulicas, dentre outros.

Partindo desta premissa, o objetivo deste estudo concentrou-se no mapeamento das chuvas intensas no estado do Tocantins com durações de 10, 30, 720 e 1440 minutos, associadas com as recorrências de 10, 50 e 100 anos, aplicando a metodologia de desagregação de chuvas e técnicas geoestatísticas. Especificamente, objetiva-se avaliar áreas críticas com relação à ocorrência de chuvas intensas, visando produzir informações úteis ao manejo e conservação do solo e da água, subsidiar o mapeamento de áreas com maior vulnerabilidade à erosão no Estado do Tocantins e produzir informação técnica para subsidiar o dimensionamento de obras hidráulicas.

\section{Materiais e Métodos}

O estudo foi realizado considerando a área do Estado do Tocantins, localizado entre os paralelos $5^{\circ} 10^{\prime} 06^{\prime \prime} \mathrm{e}$ $13^{\circ} 27^{\prime} 59^{\prime \prime}$ de latitude sul, e entre os meridianos $45^{\circ} 44^{\prime} 46^{\prime \prime}$ e 504'33" de longitude oeste, com área de $277.621 \mathrm{~km}^{2}$, representando $3,26 \%$ da área total do Brasil e 7,2\% da região norte (SEPLAN, 2013).

Segundo Souza (2019), ocorrem no Estado do Tocantins três regiões climáticas homogêneas, sendo: 1) região climática C1s2A'a': Clima subúmido seco, com grande concentração de chuvas no verão, megatérmico; 2) região climática C2wA'a': clima subúmido, com deficiência de água moderada no inverno, megatérmico e 3) região climática B1wA'a': clima úmido, com deficiência d'água moderada no inverno, megatérmico.

O banco de dados aplicado ao presente estudo consiste de séries históricas de precipitação máxima diária anual do período de 1983 a 2013 para 95 estações pluviométricas. As séries históricas foram obtidas junto ao Sistema de Informações Hidrológicas (Hidroweb) da Agência Nacional de Águas (ANA, 2015), sendo 61 postos pluviométricos localizados dentro dos limites do Estado do 
Tocantins e 34 em suas proximidades, conforme apresenta a Fig. 1. A utilização de postos localizados nas proximidades do estado visa melhorar o mapeamento na região de contorno, e também, para a adequada representação da estrutura de dependência espacial da variável (Alves et al., 2016).

Ajustou-se a distribuição de probabilidades de valores extremos máximos Gumbel para modelar a frequência das chuvas intensas diárias no Estado do Tocantins. Esta distribuição vem sendo bastante aplicada a séries de chuvas intensas, com bons resultados, podendo-se destacar os trabalhos desenvolvidos por Silva et al. (2002), Mello et al. (2001), Cardoso et al. (1998), Back (2009), Back et al. (2012), Damé et al. (2006), Damé et al. (2010) e Caldeira et al. (2016), justificando a aplicação deste modelo probabilístico no presente estudo. Visando checar a adequabilidade da distribuição Gumbel, foi aplicado o teste Kolmogorov-Smirnov ao nível de 5\% de significância.

$\mathrm{Na}$ sequência apresenta-se a descrição da distribuição Gumbel de acordo com Mello e Silva (2013). A Função Densidade de Probabilidade (FDP) da distribuição Gumbel é dada por:

$$
f(\mathrm{x})=\alpha \cdot \mathrm{e}^{\left[-\alpha \cdot(\mathrm{x}-\mu)-\mathrm{e}^{-\alpha \cdot(\mathrm{x}-\mu)}\right]}
$$

em que $x$ é a variável hidrológica em estudo, $\alpha$ é o parâ- metro de escala da distribuição e $\mu$ é o parâmetro de localização da distribuição. $\mathrm{O}$ cálculo dos parâmetros está apresentado na sequência:

$$
\begin{gathered}
\alpha=\frac{1,2826}{\mathrm{~s}} \\
\mu=\bar{x}-0,45 \cdot s
\end{gathered}
$$

em que $\bar{x}$ e $s$ correspondem à média e ao desvio padrão da série histórica, respectivamente.

A integração da FDP fornece a função cumulativa de probabilidade ( $\mathrm{FCP})$, que na forma de excedência ( $\mathrm{P}(\mathrm{X} \geq$ $\mathrm{x})$ ), é dada por:

$$
\mathrm{P}(\mathrm{X} \geq \mathrm{X})=1-\mathrm{e}^{-\mathrm{e}^{-\alpha \cdot(\mathrm{x}-\mu)}}
$$

A estimativa da variável hidrológica associada a uma dada recorrência $\left(\mathrm{X}_{\mathrm{TR}}\right)$ é dada por:

$$
x_{T R}=\frac{-L N\left[-L N\left(1-\frac{1}{T R}\right)\right]}{\alpha}+\mu
$$

em que, TR é o tempo de retorno, em anos.

A
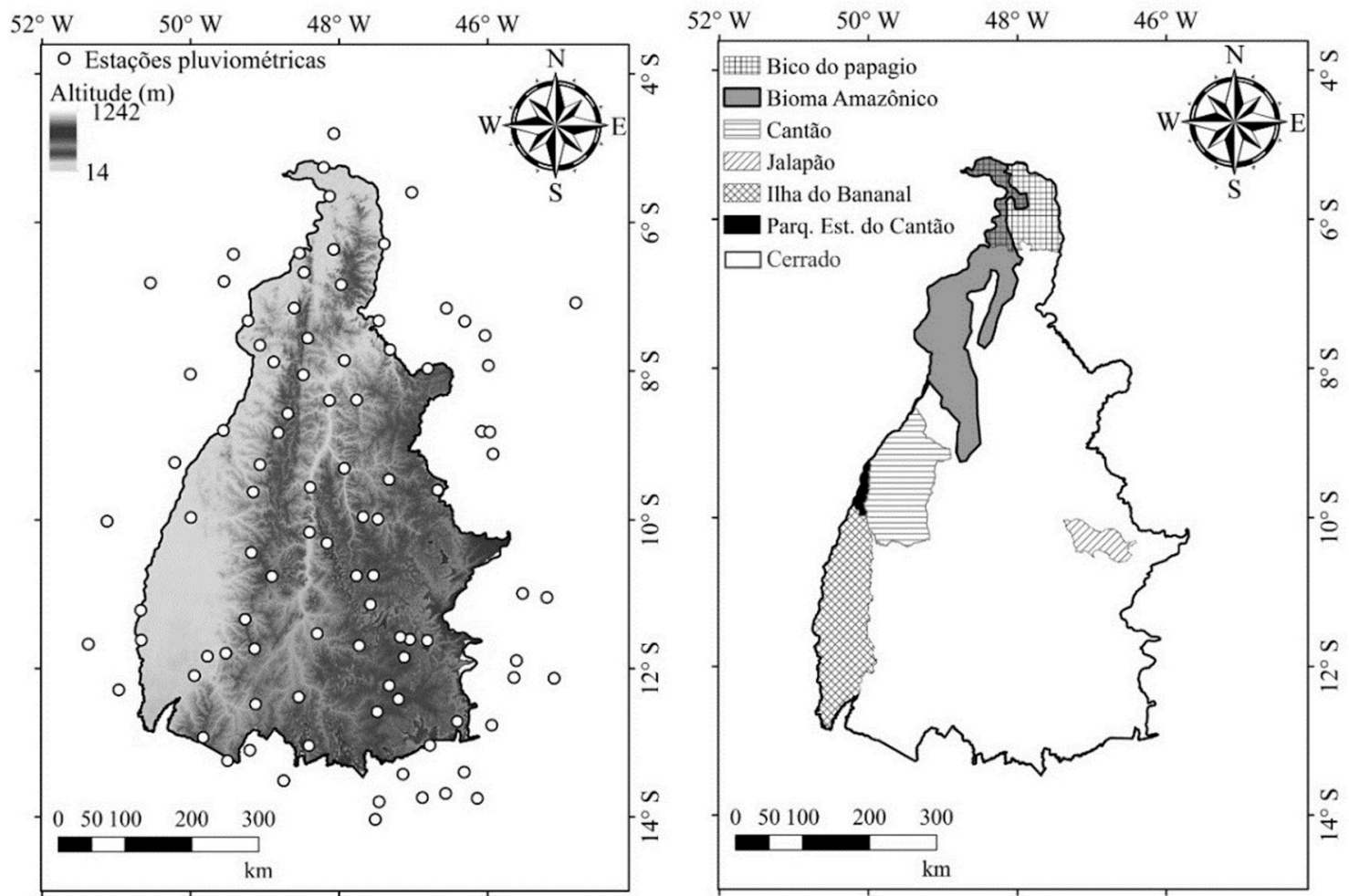

Figura 1 - Modelo digital de elevação ASTER (https://earthexplorer.usgs.gov/) e localização dos postos pluviométricos utilizados (A) e localização dos biomas Amazônia e Cerrado e principais regiões ecológicas do Tocantins (B). 
Foram aplicadas as seguintes constantes de desagregação para o Estado do Tocantins: $\mathrm{h} 24_{\mathrm{h}} / \mathrm{h}_{\mathrm{dia}}=1,14$ $\left(\right.$ CETESB, 1980), h12 $/ \mathrm{h} 24_{\mathrm{h}}=0,93, \mathrm{~h}_{1 \mathrm{~h}} / \mathrm{h}_{24 \mathrm{~h}}=0,61$, $\mathrm{h} 30_{\min } / \mathrm{h} 1_{\mathrm{h}}=0,68, \mathrm{~h} 10_{\min } / \mathrm{h} 30_{\min }=0,46$ (Silva Neto et al., 2017). Desta maneira, obtiveram-se por desagregação as chuvas intensas com durações de 1440, 720, 30 e 10 minutos, que foram associadas aos tempos de retorno de 10 , 50 e 100 anos, totalizando 12 análises.

Como procedimento inicial para a análise geoestatística, foi realizada a análise exploratória dos dados, a partir de gráficos de tendência e histogramas de distribuição de frequências para todos os eventos analisados. O primeiro com o objetivo de verificar a existência de tendência no conjunto de dados, e o segundo, a normalidade da diferença entre pares de pontos. Tais constatações prévias são essenciais para se averiguar a aplicabilidade das técnicas geoestatísticas (Ávila et al., 2009; Alves et al., 2016).

Uma etapa essencial da análise geoestatística consiste no ajuste do modelo de semivariograma teórico, obtendo-se valores para os parâmetros que estruturam tais modelos (efeito pepita, contribuição e alcance). Neste estudo, foram avaliados os modelos de semivariograma esférico, exponencial e gaussiano, tendo sido aplicado o método de ajuste dos Mínimos Quadrados Ponderados (MQP). Nas Eqs. (6)-(7)-(8) estão apresentados os modelos de semivariograma esférico, exponencial e gaussiano, respectivamente.

$$
\begin{array}{r}
\gamma(\mathrm{h})=\mathrm{C}_{0}+\mathrm{C}_{1} \cdot\left[\frac{3}{2} \cdot\left(\frac{\mathrm{h}}{\mathrm{a}}\right)-\frac{1}{2} \cdot\left(\frac{\mathrm{h}}{\mathrm{a}}\right)^{3}\right], \\
\text { se } 0<h<a ; \gamma(h)=C 0+C 1 ; h \geq a \\
\quad \gamma(\mathrm{h})=\mathrm{C}_{0}+\mathrm{C}_{1} \cdot\left[1-\exp \left(\frac{-3 \cdot \mathrm{h}}{\mathrm{a}}\right)\right], \\
\text { se } 0<h\langle a ; \gamma(h)=C 0+C 1 ; h\rangle a \\
\gamma(\mathrm{h})=C_{0}+C_{1} \cdot\left\{1-\exp \left[-3 .\left(\frac{\mathrm{h}}{\mathrm{a}}\right)^{2}\right]\right\}, \\
\text { se } 0<h\langle a ; \gamma(h)=C 0+C 1 ; h\rangle a
\end{array}
$$

sendo $\gamma(\mathrm{h})$ a semivariância, $C_{1}$ a contribuição do semivariograma (diferença entre o patamar e o efeito pepita), $a$ o alcance, $C_{0}$ o efeito pepita e $h$ a distância entre os pares de pontos.

Com o intuito de analisar a estrutura de dependência espacial dos modelos de semivariograma ajustados foi calculado o grau de dependência espacial $(G D)$, sendo obtido conforme Cambardella et al. (1994) por:

$$
G D=\left(\frac{C_{1}}{C_{0}+C_{1}}\right) \cdot 100
$$

Considerando o $G D$, pode-se adotar a seguinte classificação: $G D<25 \%$ (fraco), entre 25 e $75 \%$ (moderado) e $>75$ $\%$ (forte) (Cambardella et al., 1994).
Visando verificar o modelo de semivariograma que se sobressaiu foi realizada a validação cruzada. Foi quantificado o Erro Médio Percentual Absoluto (EMPA), possibilitando a identificação do modelo que produziu o menor erro de estimativa, e que será definido para o mapeamento de cada evento em análise. Procedimento análogo foi adotado em diversos estudos de mesma natureza, com destaque para o trabalho desenvolvido por Mello et al. (2005). O EMPA, em \%, foi calculado utilizando a seguinte expressão:

$$
E M P A=\frac{1}{n} \cdot \sum_{i=1}^{n}\left|\frac{O b s_{i}-E s t_{i}}{O b s_{i}}\right| .100
$$

em que $n$ é o número de postos pluviométricos, $O b s_{i}$ é a precipitação observada para o posto $i$ e Est $t_{i}$ é a precipitação estimada por krigagem para a posição do posto $i$.

$\mathrm{O}$ viés estatístico (Bias) foi calculado pela média da diferença entre as estimativas e as observações, indicando o erro sistemático do interpolador (subestimativa ou superestimativa). O valor do Bias corresponde ao percentual de viés das variáveis estimadas com relação às variáveis observadas. O Bias, em \%, foi obtido pela Eq. (11), de acordo com Liew et al. (2007):

$$
B I A S=\sum_{i=1}^{n}\left(O b s_{i}-E s t_{i}\right) \cdot(100) / \sum_{i=1}^{n}\left(O b s_{i}\right)
$$

Através da aplicação da krigagem ordinária, foram gerados mapas da precipitação máxima diária anual e de chuvas intensas com durações de 10, 30, 720 e 1440 minutos, associadas aos tempos de retorno de 10, 50 e 100 anos, utilizando o software ArcGIS9.2 ${ }^{\circledR}$ (ESRI, 2004). Foi adotado o sistema de Coordenadas SIRGAS 2000 para a elaboração dos mapas.

\section{Resultados e Discussão}

A partir do teste de adequação estatística de Kolmogorov-Smirnov, verificou-se que a FDP Gumbel para as 95 séries de precipitação máxima diária anual foi adequada, confirmando a aplicabilidade desta distribuição no estudo das chuvas intensas, em consonância com os resultados obtidos por Silva et. al. (2003), Souza et al. (2012) e Mello e Viola (2013). Silva et al. (2003), ao analisarem séries de precipitação máxima de diferentes durações para 10 localidades do Estado do Tocantins, também verificaram que a FDP Gumbel propiciou ajustes adequados. Apresentam-se na Tabela 1 os parâmetros dos modelos de semivariograma (efeito pepita, contribuição e alcance), o Bias, erro médio percentual absoluto e o grau de dependência espacial obtido pelos modelos de semivariograma esférico, exponencial e gaussiano, para a precipitação máxima diária anual no Estado do Tocantins. Na Fig. 2 apresenta-se os modelos teóricos exponencial, esférico e gaussiano, ajustados ao semivariograma experimental. 
Tabela 1 - Parâmetros dos modelos de semivariograma exponencial, esférico e gaussiano (efeito pepita $\left(\mathrm{C}_{0}\right)$, contribuição $\left(\mathrm{C}_{1}\right)$, alcance $(A)$ ), grau de dependência espacial (GD), Bias e erro médio percentual absoluto (EMPA) obtido por validação cruzada para a precipitação máxima diária anual no Tocantins.

\begin{tabular}{|c|c|c|c|c|c|c|}
\hline Modelo & GD (\%) & $\mathrm{C}_{0}$ & $\mathrm{C}_{1}$ & $\mathrm{~A}(\mathrm{~km})$ & Bias (\%) & EMPA (\%) \\
\hline Esférico $^{*}$ & 100,00 & 0,00 & 0,07 & 78,64 & 0,26 & 5,59 \\
\hline Exponencial & 100,00 & 0,00 & 0,07 & 99,01 & 0,34 & 5,63 \\
\hline Gaussiano & 87,98 & 4,58 & 0,06 & 66,28 & 0,24 & 5,62 \\
\hline
\end{tabular}

*Modelo considerado de melhor ajuste

Embora tenha sido encontrada pequena diferença nos valores do EMPA, o menor erro foi produzido pelo modelo esférico $(5,59 \%)$, sendo que, todos os modelos apresentaram Bias inferior a $1 \%$, alcance superior a $66 \mathrm{~km}$ e GD que os classificam como de forte dependência espacial. Souza et al. (2019) obtiveram melhor desempenho do modelo esférico em estudos sobre regionalização climática no Tocantins. Mello et al. (2003), comparando os interpoladores krigagem e inverso do quadrado da distância, também observaram bom ajuste do modelo esférico. Carvalho e Assad (2005) em estudo sobre a precipitação média anual no Estado de São Paulo, concluíram que o modelo esférico foi o que melhor se ajustou aos dados.

Na Fig. 3 está apresentado o mapa de precipitação máxima diária anual gerado por krigagem ordinária, a partir dos parâmetros do modelo de semivariograma esférico. Observa-se que as regiões do Bico do Papagaio (norte), Ilha do Bananal (noroeste, que abrange o Parque Nacional do Araguaia e Terra Indígena do Araguaia) e o centro-leste do estado podem ser consideradas críticas com relação à ocorrência da precipitação máxima diária anual. Por outro lado, na região sudeste, nas proximidades com as divisas com os estados da Bahia e Goiás, verificam-se as menores intensidades da precipitação máxima diária anual.

Outro aspecto relevante é que os valores mapeados corroboram com os estudos de Alves (2016) e Silva Neto et al. (2016). O primeiro, a partir da análise e elaboração do mapa da distribuição espacial da precipitação máxima diária anual da bacia do rio Formoso (sudoeste do Tocan-

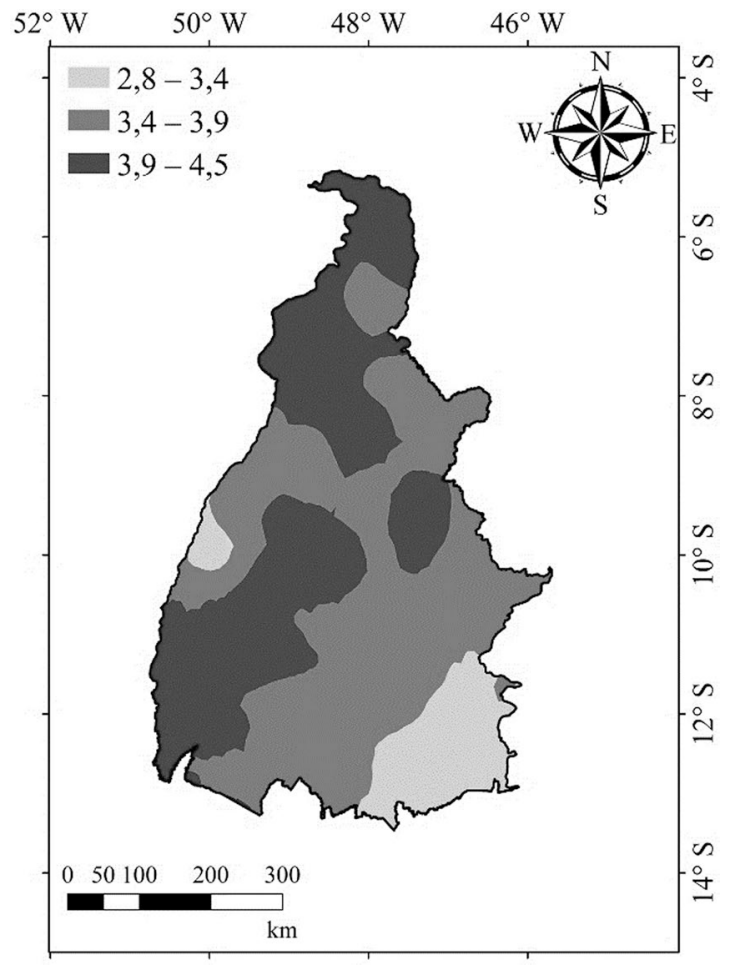

Figura 3 - Precipitação máxima diária anual $\left(\mathrm{mm} \mathrm{h}^{-1}\right)$ para o Estado do Tocantins obtido por krigagem ordinária.

tins), para o qual foi encontrada reduzida variabilidade espacial, com intensidades variando entre $3,6 \mathrm{~mm} \mathrm{~h}^{-1} \mathrm{e}$ $4 \mathrm{~mm} \mathrm{~h}^{-1}$. Já o segundo trabalho, para região Central do
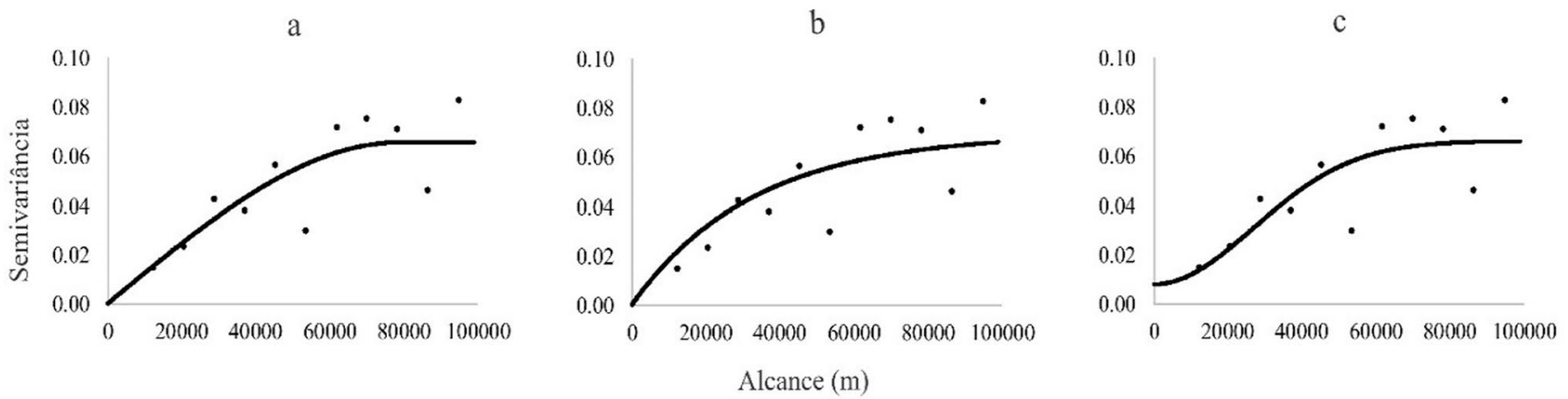

Figura 2 - Semivariogramas teóricos ajustados para o mapeamento da precipitação máxima diária anual no Tocantins. (a), esférico, (b) exponencial e (c) gaussiano. 
estado, no município de Guaraí, os autores encontram intensidades em torno de $3,6 \mathrm{~mm} \mathrm{~h}^{-1}$.

Os parâmetros dos modelos teóricos de semivariograma ajustados aos dados de chuvas intensas de 10, 30, 720 e 1440 minutos associdas com as recorrências de 10 , 50 e 100 anos, o erro médio percentual absoluto e o grau de dependência espacial estão apresentados na Tabela 2. A
Fig. 4 apresenta os modelos teóricos ajustados ao semivariograma experimental.

É possível observar na Fig. 4, a aderência dos modelos teóricos de semivariograma ajustados aos semivariogramas experimentais, com destaque para o modelo gaussiano, que no procedimento de validação cruzada, apresentou os menores erros médios percentuais nas 36

Tabela 2 - Parâmetros dos modelos de semivariograma exponencial, esférico e gaussiano (efeito pepita $\left(\mathrm{C}_{0}\right)$, contribuição $\left(\mathrm{C}_{1}\right)$, alcance $(A)$ ), grau de dependência espacial (GD), Bias e erro médio percentual absoluto (EMPA) obtido por validação cruzada para chuvas intensas com durações de 10, 30 , 720 e 1440 minutos e recorrências de 10, 50 e 100 anos.

\begin{tabular}{|c|c|c|c|c|c|c|c|}
\hline Duração (min) & Modelo & $G D(\%)$ & $C_{0}$ & $C_{1}$ & $A(\mathrm{~km})$ & Bias $(\%)$ & $E M P A(\%)$ \\
\hline \multicolumn{8}{|c|}{$\mathrm{TR}=10$ anos } \\
\hline \multirow[t]{3}{*}{10} & Esférico & 54,1 & 91,9 & 108,7 & 535 & 0,96 & 8,10 \\
\hline & Exponencial & 62,9 & 80,2 & 136,3 & 790 & 0,95 & 8,22 \\
\hline & Gaussiano $^{*}$ & 46,5 & 205,7 & 179,1 & 453 & 0,99 & 7,98 \\
\hline \multirow[t]{3}{*}{30} & Esférico & 54,1 & 91,9 & 108,7 & 535 & 0,96 & 8,10 \\
\hline & Exponencial & 62,9 & 80,2 & 136,3 & 790 & 0,95 & 8,22 \\
\hline & Gaussiano & 46,5 & 107,3 & 93,5 & 453 & 0,99 & 7,98 \\
\hline \multirow[t]{3}{*}{720} & Esférico & 54,1 & 0,8 & 0,9 & 537 & 0,96 & 8,10 \\
\hline & Exponencial & 62,9 & 0,7 & 1,1 & 795 & 0,95 & 8,22 \\
\hline & Gaussiano $^{*}$ & 46,5 & 0,9 & 0,8 & 455 & 0,99 & 7,98 \\
\hline \multirow[t]{4}{*}{1440} & Esférico & 54,1 & 0,2 & 0,2 & 537 & 0,96 & 8,11 \\
\hline & Exponencial & 62,9 & 0,2 & 0,3 & 777 & 0,95 & 8,23 \\
\hline & Gaussiano $^{*}$ & 46,5 & 0,2 & 0,2 & 456 & 0,99 & 7,98 \\
\hline & & & $\mathrm{TR}=5$ & & & & \\
\hline \multirow[t]{3}{*}{10} & Esférico & 38,41 & 488,4 & 304,5 & 402 & 1,44 & 10,03 \\
\hline & Exponencial & 45,59 & 450,1 & 377,1 & 542 & 1,44 & 10,12 \\
\hline & Gaussiano $^{*}$ & 32,37 & 540,8 & 258,8 & 364 & 1,47 & 10,00 \\
\hline \multirow[t]{3}{*}{30} & Esférico & 38,41 & 255,1 & 159,0 & 402 & 1,44 & 10,03 \\
\hline & Exponencial & 45,59 & 235,0 & 196,9 & 542 & 1,44 & 10,12 \\
\hline & Gaussiano $^{*}$ & 32,37 & 282,4 & 135,1 & 364 & 1,47 & 10,00 \\
\hline \multirow[t]{3}{*}{720} & Esférico & 38,47 & 2,21 & 1,38 & 402 & 1,44 & 10,03 \\
\hline & Exponencial & 45,67 & 2,03 & 1,71 & 542 & 1,43 & 10,11 \\
\hline & Gaussiano $^{*}$ & 32,42 & 2,44 & 1,17 & 364 & 1,47 & 9,99 \\
\hline \multirow[t]{4}{*}{1440} & Esférico & 38,33 & 0,64 & 0,40 & 401 & 1,44 & 10,02 \\
\hline & Exponencial & 45,56 & 2,59 & 0,49 & 536 & 1,43 & 10,11 \\
\hline & Gaussiano $^{*}$ & 32,30 & 0,70 & 0,34 & 363 & 1,46 & 9,99 \\
\hline & & & $\mathrm{TR}=1$ & & & & \\
\hline \multirow[t]{3}{*}{10} & Esférico & 34,4 & 680,6 & 357,6 & 388 & 1,63 & 10,66 \\
\hline & Exponencial & 40,2 & 643,5 & 433,9 & 530 & 1,63 & 10,72 \\
\hline & Gaussiano $^{*}$ & 28,9 & 741,8 & 302,8 & 349 & 1,63 & 10,61 \\
\hline \multirow[t]{3}{*}{30} & Esférico & 34,4 & 355,5 & 186,7 & 388 & 1,63 & 10,66 \\
\hline & Exponencial & 40,2 & 336,1 & 226,6 & 530 & 1,63 & 10,72 \\
\hline & Gaussiano $^{*}$ & 28,9 & 387,5 & 158,1 & 349 & 1,63 & 10,61 \\
\hline \multirow[t]{3}{*}{720} & Esférico & 34,4 & 3,0 & 1,6 & 388 & 1,63 & 10,66 \\
\hline & Exponencial & 40,2 & 2,9 & 1,9 & 530 & 1,63 & 10,72 \\
\hline & Gaussiano $^{*}$ & 28,9 & 3,3 & 1,3 & 349 & 1,63 & 10,61 \\
\hline \multirow[t]{3}{*}{1440} & Esférico & 34,3 & 0,8 & 0,4 & 388 & 1,63 & 10,66 \\
\hline & Exponencial & 40,1 & 0,8 & 0,5 & 530 & 1,63 & 10,72 \\
\hline & Gaussiano $^{*}$ & 28,9 & 0,9 & 0,3 & 349 & 1,63 & 10,61 \\
\hline
\end{tabular}

${ }^{*}$ Modelo considerado de melhor ajuste. 


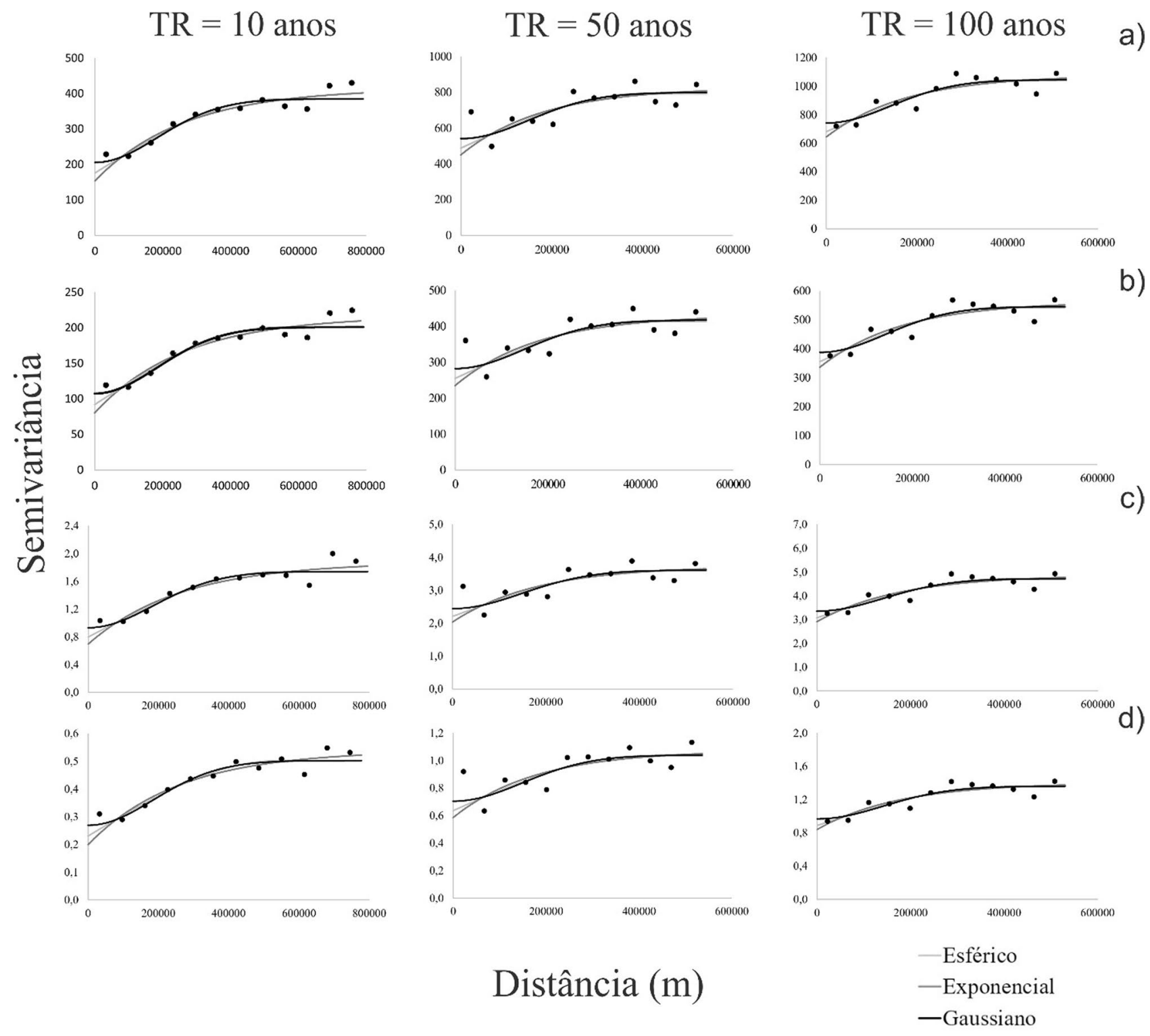

Figura 4 - Modelos de semivariograma esférico, exponencial e gaussiano ajustados para as chuvas intensas de 10 (a), 30 (b), 720 (c) e 1440 (d) minutos, com recorrências de 10, 50, e 100 anos para o Estado do Tocantins.

situações analisadas. Outro aspecto relevante considerando-se um mesmo modelo de semivariograma e TR, é que independentemente do tempo de duração da chuva, os valores de EMPA, Bias, GD e alcance são muito próximos. Isto pode ser explicado pela característica do banco de dados utilizado, que foi obtido pela metodologia de desagregação de chuvas. Nessa metodologia, inicialmente, calcula-se a chuva intensa diária associada com o TR a partir da aplicação de uma distribuição de probabilidades. Posteriormente, as chuvas intensas de menor duração e mesma recorrência são calculadas multiplicando-se o valor inicial por uma constante de desagregação. Isto explica a semelhança da estrutura de dependência espacial para um mesmo modelo de semivariograma e TR, independentemente da duração.

É importante discutir o alcance do semivariograma, pois segundo Mello e Viola (2013), o mesmo está associado à existência da dependência espacial e aplicação dos princípios da autocorrelação espacial. Considerando apenas o alcance do modelo selecionado como de melhor desempenho (gaussiano), observam-se valores que variaram de 349 a $456 \mathrm{~km}$, evidenciando abrangente estrutura de dependência espacial e consistência do banco de dados utilizados na modelagem. O Bias foi inferior a $2 \%$ e o GD apresentou-se na classe "moderado" (entre $25 \%$ e $75 \%$ ) para todas as análises. 
A Fig. 5 apresenta os mapas de chuvas intensas com durações de 10, 30, 720 e 1440 minutos associadas aos tempos de retorno de 10, 50 e 100 anos, obtidos por kriga- gem ordinária, para o estado do Tocantins. As intensidades estão apresentadas em $\mathrm{mm} \cdot \mathrm{h}^{-1}$.
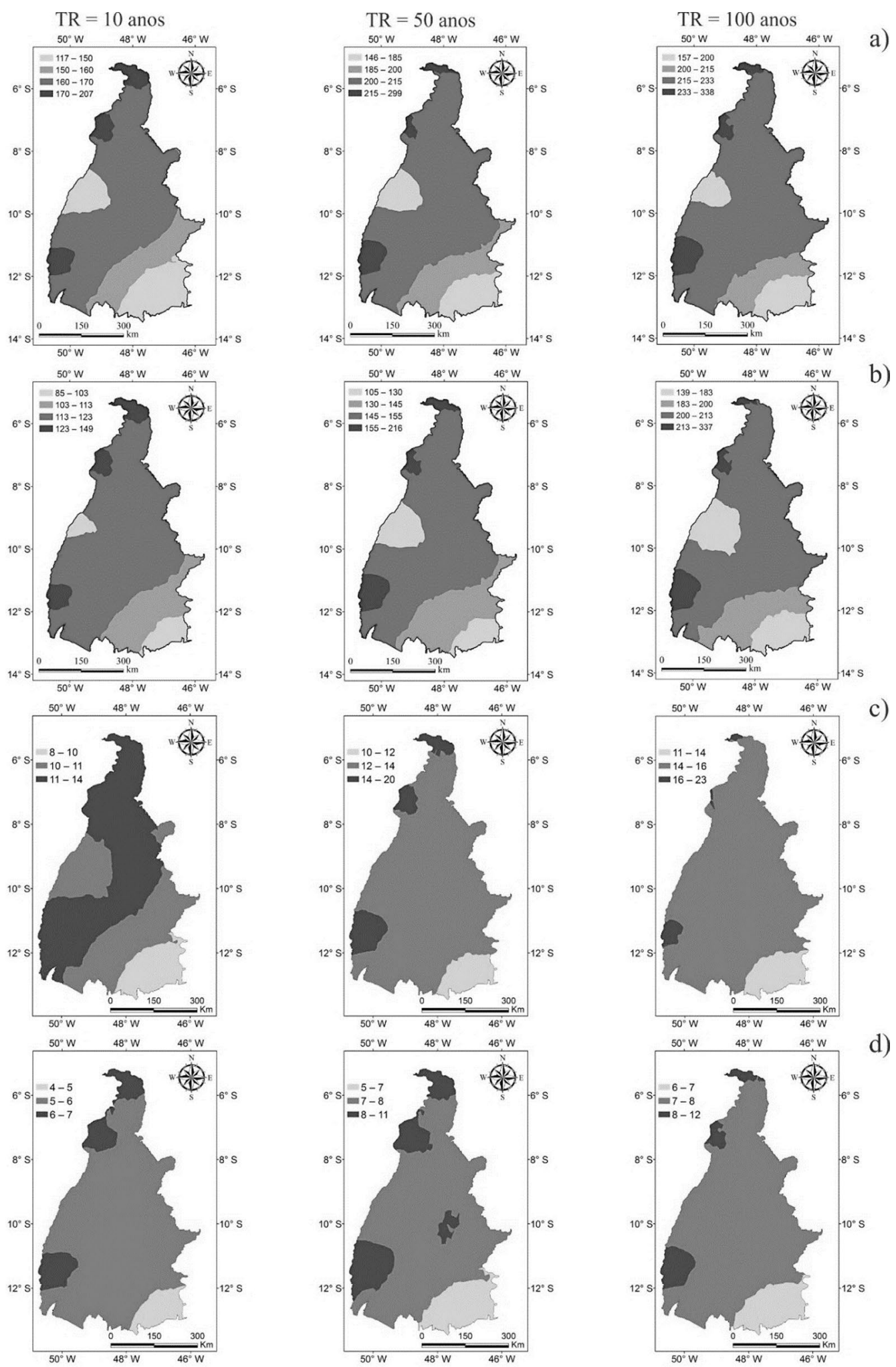

Figura 5 - Mapas de chuvas intensas com durações de 10 (a), 30 (b), 720 (c) e 1440 (d) minutos, associadas aos tempos de retorno de 10, 50 e 100 anos, em $\mathrm{mm} \mathrm{h}^{-1}$, para o Estado do Tocantins. 
Pela Fig. 5, analisando-se as chuvas intensas de curta duração (10 e $30 \mathrm{~min}$ ), observam-se altas intensidades em todo o estado. Especificamente para a chuva de $30 \mathrm{~min}$ associada ao TR de 100 anos, observam-se intensidades entre 139 e $337 \mathrm{~mm} \mathrm{~h}^{-1}$ para o Tocantins, enquanto que para o estado de Minas Gerais, conforme estudo de Mello e Viola (2013), as intensidades foram de 91,7 a 162,4 $\mathrm{mm} \mathrm{h}^{-1}$. No que tange a distribuição espacial das chuvas intensas de curta duração é possível identificar três regiões de maior magnitude, sendo a região do Bico do Papagaio (extremo norte), uma pequena porção do noroeste (logo abaixo do Bico do Papagaio), e a Ilha do Bananal (sudoeste). Estas regiões, encontram-se na divisa com os estados do Pará e Mato Grosso, e nas proximidades da área de ocorrência do bioma Amazônia, conforme pode ser visualizado na Fig. 1. Em locais nos quais prevalece este bioma há atuação marcante da massa de ar Equatorial Continental, quente e úmida, favorecendo a ocorrência de eventos de precipitação convectiva (Reboita et al., 2010), o que pode explicar os valores críticos de chuvas intensas de curta duração, quando comparado às demais regiões do estado do Tocantins.

Já para a duração intermediária avaliada (720 min) observam-se intensidades de até 14,20 e $23 \mathrm{~mm} \mathrm{~h}^{-1}$ para as recorrências de 10, 50 e 100 anos. Para esta duração, novamente verifica-se que as intensidades calculadas para o Tocantins são elevadas, quando comparado a outras regiões do Brasil. Para a chuva intensa de $720 \mathrm{~min}$ com TR de 100 anos, Mello e Viola (2013) encontraram intensidades entre 7,7 e 17,2 $\mathrm{mm} \mathrm{h}^{-1}$, para o estado de Minas Gerais. De maneira geral, a distribuição espacial desta duração (720 min) acompanha o comportamento das chuvas intensas de curta duração (10 e 30 min), com excessão do TR de 10 anos, para o qual forma-se uma faixa que liga o norte ao sudoeste do estado, passando por sua região central.

Para a maior duração trabalhada (1440 min), obtiveram-se intensidades de até $7(\mathrm{TR}=10$ anos $), 11$ ( $\mathrm{TR}=$ 50 anos) e $12 \mathrm{~mm} \mathrm{~h}^{-1}$ ( $\mathrm{TR}=100$ anos). Novamente observa-se que os valores são substancialmente superiores às chuvas calculadas para Minas Gerais por Mello e Viola (2013), que foram de até $8,8 \mathrm{~mm} \mathrm{~h}^{-1}(\mathrm{TR}=50$ anos) e de até $9,9 \mathrm{~mm} \mathrm{~h}^{-1}(\mathrm{TR}=100$ anos $)$. Chuvas de longa duração são especialmente importantes no contexto da determinação da vazão de projeto em grandes bacias hidrográficas, dado seu maior tempo de concentração. Desta maneira, evidencia-se que também para esta situação as chuvas intensas no estado do Tocantins podem ser consideradas críticas em comparação a outras localidades do Brasil. Nota-se também na Fig. 5, que as regiões sudeste e centrooeste apresentaram as menores intensidades, mas que entretanto, conforme discutido anteriormente, são elevadas quando comparadas a outras regiões brasileiras, conforme também constatado por Viola et al. (2014) e Trindade et al. (2016).
A avaliação da erosividade da chuva é tradicionalmente realizada pelo índice $\mathrm{EI}_{30}$, o qual é calculado pelo produto da energia cinética total da chuva pela sua intensidade máxima de 30 minutos (Wischmeier \& Smith, 1978). Este índice é amplamente estudado no contexto de sua aplicação na equação universal de perdas de solo (USLE). A classificação do índice $\mathrm{EI}_{30}$, conforme Foster et al. (1981), segue a seguinte distribuição: $\mathrm{EI}_{30} \leq 2.452$ (erosividade fraca), $2.452<\mathrm{EI}_{30} \leq 4.905$ (moderada), $4.905<$ $\mathrm{EI}_{30} \leq 7.357$ (moderada a forte), $7.357<\mathrm{EI}_{30} \leq 9.810$

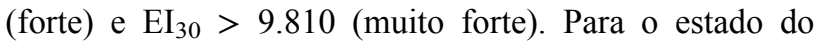
Tocantins, Viola et al. (2014) encontraram valores de $\mathrm{EI}_{30}$ entre 6.599 e $14.000 \mathrm{MJ} \mathrm{mm} \mathrm{ha}^{-1} \mathrm{~h}^{-1}$ ano $^{-1}$, situando-se entre as classes de moderada a forte e muito forte. Tais resultados encontram respaldo aos obtidos no presente estudo no contexto de chuvas intensas, ou seja, regiões com predominância de chuvas mais intensas também apresentam o maior potencial erosivo.

\section{Conclusões}

O modelo de semivariograma esférico apresentou melhor ajuste para o mapeamento da precipitação máxima diária anual, enquanto que para as chuvas intensas calculadas pela metodologia de desagregação de chuvas houve melhor desempenho do modelo gaussiano.

As chuvas intensas de diferentes durações apresentam altas intensidades no estado do Tocantins, quando comparado a outras regiões brasileiras. As regiões do Bico do Papagaio (norte), noroeste e Ilha do Bananal (sudoeste) apresentam ocorrência crítica no contexto estadual.

O mapeamento das chuvas intensas de curta duração, especialmente a de $30 \mathrm{~min}$, associado a estudos prévios sobre a erosividade da chuva no estado do Tocantins permite concluir que o desenvolvimento de pesquisas sobre a erosão hídrica como sendo prioritário para o planejamento agropecuário estadual.

\section{Agradecimentos}

À Agência Nacional de Águas (ANA) pela disponibilização de séries hidrológicas, ao Instituto Federal do Tocantins pela concessão de bolsa de qualificação - PROQUALIFICAR, Edital n ${ }^{\circ}$ 24/2016 e ao o CNPq pela concessão de bolsa produtividade em pesquisa. In memorian ao saudoso professor Silvio

\section{Referências}

ALVES, T.L.B.; DE AZEVEDO, P.V.; DE FARIAS, A.A. Distribuição da precipitação mensal, anual e máxima diária anual na bacia hidrográfica do rio Formoso, Tocantins. Ambiência, v. 12, n. 1, p. 49-70, 2016.

ANA. Agência Nacional das Águas. Hidroweb. Sistema de informações hidrológicas. Disponível em: < http://hidro web.ana.gov.br>. Acesso em: 10 de maio de 2015. 
ARAGÃO, R.D.; SANTANA, G.R.D.; COSTA, C.E.F.F.D.; CRUZ, M.A.S.; FIGUEIREDO, E.E.D.; SRINIVASAN, V.S. Chuvas intensas para o estado de Sergipe com base em dados desagregados de chuva diária. Revista Brasileira de Engenharia Agrícola e Ambiental, v. 17, n. 3, p. 243-252, 2013.

ÁVILA, L.F.; MELLO, C.D.; VIOLA, M.R. Mapeamento da precipitação mínima provável para o sul de Minas Gerais. Revista Brasileira de Engenharia Agrícola e Ambiental, v. 13, (Suplemento), p. 906-915, 2009.

BACK, Á.J.; HENN, A.; OLIVEIRA, J.L.R. Heavy rainfall equations for Santa Catarina, Brazil. Revista Brasileira de Ciência do Solo, v. 35, p. 2127-2134, 2011.

BACK, Á.J.; OLIVEIRA, J.L.R.; HENN, A. Relações entre precipitações intensas de diferentes durações para desagregação da chuva diária em Santa Catarina. Revista Brasileira de Engenharia Agrícola e Ambiental, v. 16, n. 4, p. 391-398, 2012.

BACK, A. Relações entre precipitações intensas de diferentes durações ocorridas no município de Urussanga, SC. Revista Brasileira de Engenharia Agrícola e Ambiental, v. 13, n. 2, p. 170-175, 2009.

CALDEIRA, T.L.; BESKOW, S.; MELLO, C.R.D.; VARGAS, M.M.; GUEDES, H.A.S.; COLL, L. F. Desagegação de chuva diária: uma análise para o Estado do Rio Grande do Sul. Revista Scientia Agraria (SA), v. 16, n. 3, p. 1-21, 2016.

CAMBARDELLA, C.; MOORMAN, T.; PARKIN, T.; KARLEN, D.; NOVAK, J.; TURCO, R.; KONOPKA, A. Fieldscale variability of soil properties in central Iowa soils. Soil science society of America journal, v. 58, n. 5, p. 15011511, 1994.

CARDOSO, C.O.; ULLMANN, M.N.; BERTOL, I. Análise de chuvas intensas a partir da desagregação das chuvas diárias de Lages e de Campos Novos (SC). Revista Brasileira de Ciência do Solo, v. 22, p. 131-140, 1998.

CARVALHO, J.R.P.; ASSAD, E.D. Análise espacial da precipitação pluviométrica no Estado de São Paulo: comparação de métodos de interpolação. Engenharia Agrícola, v. 25, n. 2, p. 377-384, 2005.

COMPANHIA DE TECNOLOGIA AMBIENTAL DO ESTADO DE SÃO PAULO - CETESB. CETESB. Drenagem Urbana: manual de projetos. São Paulo: DAEE/ CETESB, 1980. 466 p.

DAMÉ, R.D.C.F.; PEDROTTI, C.B.M.; CARDOSO, M.A.G.; SILVEIRA, C.P.D.; DUARTE5, L.A.; ÁVILA, M. S.V.D.; MOREIRA, A. Comparação entre curvas intensidade-duração-frequência de ocorrência de precipitação obtidas a partir de dados pluviográficos com aquelas estimadas por técnicas de desagregação de chuva diária. Revista Brasileira de Agrociência, v. 12, n. 4, p. 505-509, 2006.

DAMÉ, R.D.C.; TEIXEIRA, C.F.; TERRA, V.S.; ROSSKOFF, J.L. Hidrograma de projeto em função da metodologia utilizada na obtenção da precipitação. Revista Brasileira de Engenharia Agrícola e Ambiental, v. 14, n. 1, p. 46-54, 2010.

ESRI - Environmental Systems Research Institute. ArcGIS 9: Getting started with ArcGIS. Redlands. 265 p., 2004.
FESTI, A.V. Coletâne de Equações de Chuvas no Brasil. Anais do XVII Simpósio Brasileiro De Recursos Hídricos. São Paulo, p. 1-18, 2007.

FOSTER, G.R.; McCOOL, D.K.; RENARD, K.G. \& MOLDENHAUER, W.C. Conversion of the universal soil loss equation to SI metric units. Journal of Soil and Water Conservation, v. 36, n. 6, p. 355-359, 1981.

LIEW, M.W.; VEITH, T.L.; BOSCH, D.D.; ARNOLD, J.G. Suitability of SWAT for the conservation effects assessment project: Comparison on USDA agricultural research service watersheds. Journal of Hydrologic Engineering, v. 12, n. 2, p. 173-189, 2007.

MARCUZZO, F.F.N.; GOULARTE, E.R.P. Índice de Anomalias de Chuvas do Estado do Tocantins. Geoambiente on line, v. 1, n. 19, p. 55-71, 2012.

MARCUZZO, F.F.N.; GOULARTE, E.R.P. Caracterização do Ano Hidrológico e Mapeamento Espacial das Chuvas nos Períodos Úmido e Seco do Estado do Tocantins. Revista Brasileira de Geografia Física, v. 6, n. 01, p. 91-99, 2013.

MELLO, C.R.; FERREIRA, D.; SILVA, A.; LIMA, J. Análise de modelos matemáticos aplicados ao estudo de chuvas intensas. Revista Brasileira de Ciência do Solo, Viçosa, v. 25, n. 3, p. 693-698, 2001

MELLO, C.R; LIMA, J.; SILVA, A.; MELLO, J.; OLIVEIRA, M. Krigagem e inverso do quadrado da distância para interpolação dos parâmetros da equação de chuvas intensas. Revista Brasileira de Ciência do Solo, v. 27, n. 5, p. 925933, 2003.

MELLO, C.R.; SILVA, A. Hidrologia: Princípios e aplicações em sistemas agrícolas. Lavras: Ed. UFLA, 455 p., 2013.

MELLO, C.R.; VIOLA, M.R. Mapeamento de chuvas intensas no estado de Minas Gerais. Revista Brasileira de Ciência do Solo, v. 37, p. 37-44, 2013.

MELLO, J.M.D.; BATISTA, J.L.F.; JÚNIOR, P.J.R.; OLIVEIRA, M.S.D. Ajuste e seleção de modelos espaciais de semivariograma visando à estimativa volumétrica de Eucalyptus grandis. Scientia Forestalis, n. 69, p. 25-37, 2005.

OLIVEIRA, L.F.C.D.; CORTÊS, F.C.; BARBOSA, F.D.O.A.; ROMÃO, P.D.A.; CARVALHO, D.F.D. Estimativa das Equações de Chuvas Intensas para algumas localidades no Estado de Goiás pelo Método de desagregação de Chuvas. Pesquisa Agropecuária Brasileira, v. 30, p. 23-27, 2000.

OLIVEIRA, L.F.C.D.; ANTONINI, J.C.D.A.; GRIEBELER, N.P. Estimativas de chuvas intensas para o Estado de Goiás. Eng. Agríc., v. 28, n. 1, p. 22-33, 2008.

PFAFSTETTER, O. Chuvas intensas no Brasil. Rio de Janeiro: Ministério da Viação e Obras púbicas; DNOS, 420 p., 1957.

REBOITA, M.S.; GAN, M.A.; ROCHA, R.P.D.; AMBRIZZI, T. Regimes de precipitacao na America do sul: uma revisão bibliográfica. Revista Brasileira de Meteorologia, v. 25, n. 2, p. 185-204, 2010.

SEPLAN. Secretária de Planejamento. Atlas do Tocantins: Subsídios ao Planejamento da Gestão Territorial. Superintendência de Planejamento e Gestão central de Políticas Públicas. Diretoria de Zoneamento EcológicoEconômico - DZE. Organizado por. BORGES, R.S.T.; DIAS, R.R.; SOUSA, P.A.B. (orgs). 6. ed. rev. atua. Palmas: Seplan, 80 p., 2012.

SILVA NETO, V.L.; VIOLA, M.R.; RÊGO, P.L.; OLIVEIRA, A.L.D.; SILVA, L.L.D. Relação Intensidade-Duração-Fre- 
quência (IDF) Para Precipitação Extrema em Guaraí-TO. Enciclopédia Biosfera, v. 13, n. 23, p. 1146-1154, 2016.

SILVA NETO, V.L.; VIOLA, M.R.; SILVA, D.D.D.; MELLO, C.R.D.; PEREIRA, S.B.; GIONGO, M. Daily rainfall disaggregation for Tocantins State, Brazil. Revista Ambiente \& Água, v. 12, n. 4, p. 605-617, 2017.

SILVA, D.D.; GOMES FILHO, R.R.; PRUSKI, F.F.; PEREIRA, S.B.; NOVAES, L.F.D. Chuvas intensas no Estado da Bahia. Revista Brasileira de Engenharia Agrícola e Ambiental, v. 6, n. 2, p. 362-367, 2002.

SILVA, D.D.; PEREIRA, S.B.; PRUSKI, F.F.; RODRIGUES, R.; FILHO, G.; et al. Equações de Intensidade-duração-frequência da precipitação pluvial para o Estado do Tocantins. Revista Engenharia na Agricultura, v. 11, n. 4, p. 7-14, 2003.

SOUZA, F.H.M.D.; VIOLA, M.R.; AVANZI, J.C.; GIONGO, M.; VIEIRA FILHO, M. Thornthwaite's Climate Regionalization for the State of Tocantins, Brazil. Floresta, v. 49, n. 4, p. 10, 2019.

SOUZA, R.O.R.D. M.; SCARAMUSSA, P.H.M.; AMARAL, M.A.C.M.D.; PEREIRA NETO, J.A.; PANTOJA, A.V.;
SADECK, L.W.R. Equações de chuvas intensas para o estado do Pará. Revista Brasileira de Engenharia Agrícola e Ambiental, v. 16, n. 9, p. 999-1005, 2012.

TRINDADE, A.L.F.; OLIVEIRA, P.T.S.D.; ANACHE, J.A.A.; WENDLAND, E. Variabilidade espacial da erosividade das chuvas no Brasil. Pesquisa Agropecuária Brasileira, v. 51, p. 1918-1928, 2016.

VIOLA, M.R.; AVANZI, J.C.; MELLO, C.R.D.; LIMA, S.D.O.; ALVES, M.V.G. Distribuição e potencial erosivo das chuvas no Estado do Tocantins. Pesquisa Agropecuária Brasileira, v. 49, p. 125-135, 2014.

WISCHMEIER, W.H.; SMITH, D.D. Predicting rainfall erosion losses a guide to conservation planning. Agriculture Handbook, U.S. Departament of Agriculture, Washington n. 537,58 p., 1978.

License information: This is an open-access article distributed under the terms of the Creative Commons Attribution License (type CC-BY), which permits unrestricted use, distribution and reproduction in any medium, provided the original article is properly cited. 